\title{
System of Equations for Antenna Array Beam Pattern Nulling
}

\author{
Alexey A. Erokhin, Yury P. Salomatov, Member, IEEE \\ Institute of Engineering Physics and Radio Electronics \\ Siberian Federal University \\ Krasnoyarsk, Russia \\ AErokhin@sfu-kras.ru
}

\begin{abstract}
Method of synthesis a beam pattern with nulls in specified directions is produced. In the synthesis of the beam pattern used a solution of the system of equations. An expression to calculate the beamformer complex weights is shown.
\end{abstract}

Keywords-antenna arrays; antenna beam patterns; adaptive array;

\section{INTRODUCTION}

The principle of interference suppression in the adaptive antenna array is based on a weighted summation of patterns. With this summation side reception in directions of interference disappears. The main lobe of the beam pattern little varies, because side lobes of the additional pattern get in this area.

\section{BAEM PATTERN NULLLING IN SPECIFIED DIRECTIONS}

As shown in $[1,2]$ expression for beam pattern with null in specified direction $\left(\theta_{1}, \varphi_{1}\right)$ has the form:

$$
\dot{F}_{\Delta 1}\left(\theta, \varphi, \theta_{0}, \varphi_{0}\right)=\dot{F}\left(\theta, \varphi, \theta_{0}, \varphi_{0}\right)-\dot{F}_{p 1} \cdot \dot{F}\left(\theta, \varphi, \theta_{1}, \varphi_{1}\right)
$$

where $\{\cdot\}$ - denote complex value, $\dot{F}\left(\theta, \varphi, \theta_{i}, \varphi_{i}\right)$ - radiation pattern of array with main lobe direction $\left(\theta_{i}, \varphi_{i}\right)$, $\dot{F}_{p 1}=\frac{\dot{F}\left(\theta_{1}, \varphi_{1}, \theta_{0}, \varphi_{0}\right)}{\dot{F}\left(\theta_{1}, \varphi_{1}, \theta_{1}, \varphi_{1}\right)}=$ const - coefficient depending on the direction of the null, with definition from $\dot{F}_{\Delta 1}\left(\theta_{1}, \varphi_{1}, \theta_{0}, \varphi_{0}\right)=0$.

Also in [1] shown that beamformer complex weights for forming this beam pattern is

$$
\dot{I}_{\Delta}=\dot{I}_{0}-\dot{F}_{p} \cdot \dot{I}_{1}
$$

where $\dot{I}_{0}$ - beamformer complex weights for undistorted beam pattern, $\dot{I}_{1}$ - beamformer complex weights for beam pattern with main lobe direction $\left(\theta_{1}, \varphi_{1}\right)$.
This method can be applied to cases with a lot of nulls. Let us show it. To form the second null in the beam pattern we can write:

$$
\dot{F}_{\Delta 2}\left(\theta, \varphi, \theta_{0}, \varphi_{0}\right)=\dot{F}_{\Delta 1}\left(\theta, \varphi, \theta_{0}, \varphi_{0}\right)-\dot{F}_{p 2} \cdot \dot{F}_{\Delta 12}\left(\theta, \varphi, \theta_{2}, \varphi_{2}\right)
$$

where $\dot{F}_{p 2}=\frac{\dot{F}_{\Delta_{1}}\left(\theta_{2}, \varphi_{2}, \theta_{0}, \varphi_{0}\right)}{\dot{F}_{\Delta 12}\left(\theta_{2}, \varphi_{2}, \theta_{2}, \varphi_{2}\right)}$,

$\dot{F}_{\Delta 12}\left(\theta, \varphi, \theta_{2}, \varphi_{2}\right)=\dot{F}\left(\theta, \varphi, \theta_{2}, \varphi_{2}\right)-\dot{F}_{p 12} \cdot \dot{F}\left(\theta, \varphi, \theta_{1}, \varphi_{1}\right) \quad$ - beam pattern with main lobe direction $\left(\theta_{2}, \varphi_{2}\right)$ and null in direction $\left(\theta_{1}, \varphi_{1}\right), \dot{F}_{p 12}=\frac{\dot{F}\left(\theta_{1}, \varphi_{1}, \theta_{2}, \varphi_{2}\right)}{\dot{F}\left(\theta_{2}, \varphi_{2}, \theta_{2}, \varphi_{2}\right)}$.

And now if we want to create the third null:

$\dot{F}_{\Delta 3}\left(\theta, \varphi, \theta_{0}, \varphi_{0}\right)=\dot{F}_{\Delta 2}\left(\theta, \varphi, \theta_{0}, \varphi_{0}\right)-\dot{F}_{p 3} \cdot \dot{F}_{\Delta 123}\left(\theta, \varphi, \theta_{2}, \varphi_{2}\right)$,

where $-\dot{F}_{p 3}=\frac{\dot{F}_{\Delta 2}\left(\theta_{3}, \varphi_{3}, \theta_{0}, \varphi_{0}\right)}{\dot{F}_{\Delta 123}\left(\theta_{3}, \varphi_{3}, \theta_{3}, \varphi_{3}\right)}$,

$\dot{F}_{\Delta 123}\left(\theta, \varphi, \theta_{3}, \varphi_{3}\right)=\dot{F}_{\Delta 13}\left(\theta, \varphi, \theta_{3}, \varphi_{3}\right)-\dot{F}_{p 123} \cdot \dot{F}_{\Delta 132}\left(\theta, \varphi, \theta_{2}, \varphi_{2}\right)$,

$\dot{F}_{p 123}=\frac{\dot{F}_{\Delta 13}\left(\theta_{2}, \varphi_{2}, \theta_{3}, \varphi_{3}\right)}{\dot{F}_{\Delta 132}\left(\theta_{2}, \varphi_{2}, \theta_{2}, \varphi_{2}\right)}$,

$\dot{F}_{\Delta 132}\left(\theta, \varphi, \theta_{3}, \varphi_{3}\right)=\dot{F}\left(\theta, \varphi, \theta_{3}, \varphi_{3}\right)-\dot{F}_{p 12} \cdot \dot{F}\left(\theta, \varphi, \theta_{1}, \varphi_{1}\right)$,

$\dot{F}_{\Delta 13}\left(\theta, \varphi, \theta_{3}, \varphi_{3}\right)=\dot{F}\left(\theta, \varphi, \theta_{3}, \varphi_{3}\right)-\dot{F}_{p 13} \cdot \dot{F}\left(\theta, \varphi, \theta_{1}, \varphi_{1}\right)$.

$\dot{F}_{p 13}=\frac{\dot{F}\left(\theta_{1}, \varphi_{1}, \theta_{3}, \varphi_{3}\right)}{\dot{F}\left(\theta_{3}, \varphi_{3}, \theta_{3}, \varphi_{3}\right)}$.

Thus, using the considered method we can get the beam pattern and the beamformer complex weights for any number of nulls; also received expressions are valid for antenna arrays with any geometry, and for continuous antennas. For continuous antennas expression for current will have the form: 


$$
\dot{I}_{\Delta}(z)=\dot{I}_{0}(z)-\dot{F}_{p} \cdot \dot{I}_{1}(z) .
$$

\section{COMPOSE A SYSTEM OF EQUATIONS}

Let us consider the beam pattern of the antenna array with $M$ nulls in specified directions as the weighted sum of beam patterns this array with the maximum of the main lobe in $M$ directions:

$$
\dot{F}(\theta, \varphi)=\sum_{m=0}^{M} \dot{\alpha}_{m} \dot{F}\left(\theta, \varphi, \theta_{m}, \varphi_{m}\right),
$$

where $\dot{F}\left(\theta, \varphi, \theta_{m}, \varphi_{m}\right)$ - beam patterns of the antenna array with the maximum in direction $\left(\theta_{m}, \varphi_{m}\right)$. Let us suppose that $m=0$ correspond to the direction of interest signal and $1 \leq m \leq M$ correspond to the direction of interference signal (nulls of pattern in this directions will be formed).

We assume that coefficient $\alpha_{0}$ is equal to some constant, according to normalization condition (like a level of beam pattern in direction of interest signal). Rest of coefficients $\alpha_{m}$ we find from this condition:

$$
F\left(\theta_{m}, \varphi_{m}\right)=0,1 \leq m \leq M
$$

This condition will help to obtain linear system of equations

$$
\left\{\begin{array}{l}
\sum_{m=0}^{M} \dot{\alpha}_{m} \dot{F}\left(\theta_{1}, \varphi_{1}, \theta_{m}, \varphi_{m}\right)=0 \\
\sum_{m=0}^{M} \dot{\alpha}_{m} \dot{F}\left(\theta_{2}, \varphi_{2}, \theta_{m}, \varphi_{m}\right)=0 \\
\vdots \\
\sum_{m=0}^{M} \dot{\alpha}_{m} \dot{F}\left(\theta_{M}, \varphi_{M}, \theta_{m}, \varphi_{m}\right)=0
\end{array}\right.
$$

We can choose coefficient $\alpha_{0}$ arbitrarily so that we can rewrite received system of equations in the following form:

$$
\left\{\begin{array}{l}
\sum_{m=1}^{M} \dot{\alpha}_{m} \dot{F}\left(\theta_{1}, \varphi_{1}, \theta_{i}, \varphi_{i}\right)=-\dot{F}\left(\theta_{1}, \varphi_{1}, \theta_{0}, \varphi_{0}\right) ; \\
\sum_{m=1}^{M} \dot{\alpha}_{m} \dot{F}\left(\theta_{2}, \varphi_{2}, \theta_{m}, \varphi_{m}\right)=-\dot{F}\left(\theta_{2}, \varphi_{2}, \theta_{0}, \varphi_{0}\right) ; \\
\vdots \\
\sum_{m=1}^{M} \dot{\alpha}_{m} \dot{F}\left(\theta_{M}, \varphi_{M}, \theta_{m}, \varphi_{m}\right)=-\dot{F}\left(\theta_{M}, \varphi_{M}, \theta_{0}, \varphi_{0}\right) ;
\end{array}\right.
$$

and this system must be supplemented by one more equation which introduced constraint on level of main lobe in the interest signal direction:

$$
\sum_{m=0}^{M} \dot{\alpha}_{m} \dot{F}\left(\theta_{0}, \varphi_{0}, \theta_{m}, \varphi_{m}\right)=C
$$

Since $\alpha_{0}=1$ :

$$
\dot{F}\left(\theta_{0}, \varphi_{0}, \theta_{0}, \varphi_{0}\right)+\sum_{m=1}^{M} \dot{\alpha}_{m} \dot{F}\left(\theta_{0}, \varphi_{0}, \theta_{m}, \varphi_{m}\right)=C
$$

If we impose the requirement distortionless on level of beam pattern in the interest signal direction then second term in previous equation:

$$
\sum_{m=1}^{M} \dot{\alpha}_{m} \dot{F}\left(\theta_{0}, \varphi_{0}, \theta_{m}, \varphi_{m}\right)=0
$$

Actually this is not possible. And it is necessary to minimize this sum:

$$
\sum_{m=1}^{M} \dot{\alpha}_{m} \dot{F}\left(\theta_{0}, \varphi_{0}, \theta_{m}, \varphi_{m}\right)=\varepsilon .
$$

where $\varepsilon<<1$.

When the beam pattern of the antenna array using only as an array factor:

$$
\dot{F}_{\Sigma}\left(\theta_{i}, \varphi_{i}, \theta_{j}, \varphi_{j}\right)=\dot{F}_{\Sigma}^{*}\left(\theta_{j}, \varphi_{j}, \theta_{i}, \varphi_{i}\right)
$$

where $\{*\}$ - denote complex conjugate. This condition leads to a specific type equations in matrix form (square matrix composed of coefficients $\dot{F}\left(\theta_{i}, \varphi_{i}, \theta_{j}, \varphi_{j}\right)$ will be a Hermitian matrix). In common case we have:

$$
\dot{F}_{\Sigma}\left(\theta_{i}, \varphi_{i}, \theta_{j}, \varphi_{j}\right) \neq \dot{F}_{\Sigma}^{*}\left(\theta_{j}, \varphi_{j}, \theta_{i}, \varphi_{i}\right)
$$
form:

After finding the coefficients $\alpha_{\mathrm{m}}$ expression (1) take a

$$
\dot{F}(\theta, \varphi)=\dot{F}\left(\theta, \varphi, \theta_{0}, \varphi_{0}\right)+\sum_{m=1}^{M} \dot{\alpha}_{m} \dot{F}\left(\theta, \varphi, \theta_{m}, \varphi_{m}\right)
$$

As $\dot{F}\left(\theta, \varphi, \theta_{i}, \varphi_{i}\right)$ we can use expression for discrete antenna array beam pattern or expression for continuous radiator beam pattern.

\section{Search Weighting CoefFicients}

In case of a linear antenna array expression for the beam pattern has the form 


$$
\dot{F}\left(\theta, \theta_{m}\right)=\sum_{n=1}^{N} \dot{I}_{n, m} \exp \left(-j k z_{n} \sin \theta\right),
$$

where $N$ - number of elements, $z_{n}$ - coordinate of $n$-th element, $\dot{I}_{n, m}$ - amplitude and phase of $n$-th element for steering main bean to direction $\theta_{m}$ (beamformer complex weights).

For equal amplitude of array elements excitation:

$$
\dot{F}\left(\theta, \theta_{m}\right)=\dot{I}_{m} \sum_{n=1}^{N} \exp \left[-j k z_{n}\left(\sin \theta-\sin \theta_{m}\right)\right] .
$$

Accordance to previous expression we can control only one parameter $\dot{I}_{m}$ (amplitude of elements) for a given $\theta_{m}$.

Rewrite the expression (5) for the linear antenna array:

$$
\dot{F}(\theta)=\dot{F}\left(\theta, \theta_{0}\right)+\sum_{m=1}^{M} \dot{\alpha}_{m} \dot{F}\left(\theta, \theta_{m}\right)
$$

And now the expression (6) has the form:

$$
\dot{F}\left(\theta, \theta_{m}\right)=\dot{I}_{m} \dot{F}_{\Sigma}\left(\theta, \theta_{m}\right)
$$

where $\dot{F}_{\Sigma}\left(\theta, \theta_{m}\right)=\sum_{n=1}^{N} \exp \left[-j k z_{n}\left(\sin \theta-\sin \theta_{m}\right)\right]$.

So that system of equations (4) can be written as:

$$
\left\{\begin{array}{l}
\sum_{m=1}^{M} \dot{\alpha}_{m} I_{m} \dot{F}_{\Sigma}\left(\theta_{1}, \theta_{m}\right)=-I_{0} \dot{F}_{\Sigma}\left(\theta_{1}, \theta_{0}\right) ; \\
\sum_{m=1}^{M} \dot{\alpha}_{m} I_{m} \dot{F}_{\Sigma}\left(\theta_{2}, \theta_{m}\right)=-I_{0} \dot{F}_{\Sigma}\left(\theta_{2}, \theta_{0}\right) ; \\
\vdots \\
\sum_{m=1}^{M} \dot{\alpha}_{m} I_{m} \dot{F}_{\Sigma}\left(\theta_{M}, \theta_{m}\right)=-I_{0} \dot{F}_{\Sigma}\left(\theta_{M}, \theta_{0}\right) ;
\end{array}\right.
$$

The multiplication of $\alpha_{0} I_{0}$ also contained in the right side of this system of equations, because previously we choose $\alpha_{0}=1$.

Suppose that after solving the system of equations (7) we found the amplitude and phase of $n$-th element $\left(\dot{I}_{n}\right)$. Then we can write the expression for beam pattern of linear array:

$$
\dot{F}(\theta)=\sum_{n=1}^{N} \dot{I}_{n} \exp \left(-j k z_{n} \sin \theta\right)
$$

Rewrite previous expression taking into account the expression (2)

$$
\sum_{n=1}^{N} \dot{I}_{n} \exp \left(-j k z_{n} \sin \theta_{m}\right)=0,1 \leq m \leq M
$$

We obtain a system of linear equations, that equivalent system of equations (3) or (4). This system has a solution only if $M<N$

Accordance with (6) we can write the beamformer complex weights:

$$
\dot{I}_{n, m}^{\prime}=\dot{I}_{m} \exp \left(j k z_{n} \sin \theta_{m}\right) .
$$

Therefore expression for the beamformer complex weights with $M$ nulls has the form:

$$
\dot{I}_{\Sigma n}=\sum_{m=0}^{M} \alpha_{m} \exp \left(j k z_{n} \sin \theta_{m}\right) .
$$

Now we can solve system of equation for uniform linear array with number of elements $N=7$. We find coefficient $\alpha$ for two cases: $M=6$ and $M=7$.

In Fig. 1 shown beam patterns for these cases:

- $\quad$ solid line $M=6\left(\theta_{0}=0^{\circ}, \theta_{1}=-50^{\circ}, \theta_{2}=-60^{\circ}\right.$, $\left.\theta_{3}=-70^{\circ}, \theta_{4}=20^{\circ}, \theta_{5}=30^{\circ}, \theta_{6}=40^{\circ}\right)$;

- dash line $\mathrm{M}=7\left(\theta_{0}=0^{\circ}, \theta_{1}=-50^{\circ}, \theta_{2}=-60^{\circ}\right.$, $\theta_{3}=-70^{\circ}, \theta_{4}=20^{\circ}, \theta_{5}=30^{\circ}, \theta_{6}=40^{\circ}, \theta_{7}=45^{\circ}$ ).

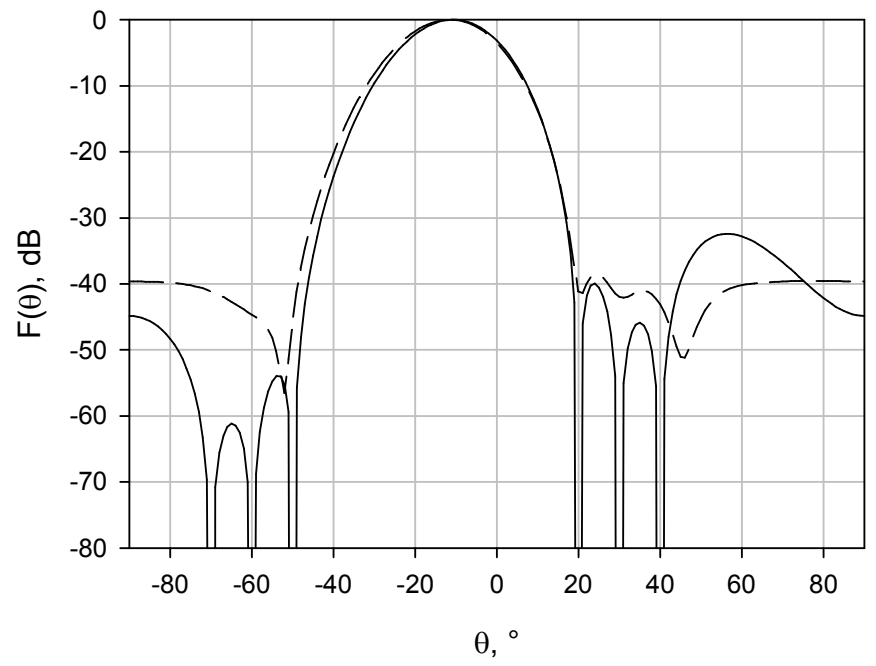

Fig. 1. Beam pattern of linear 7-element array.

\section{SUMMARY}

It was shown that the principle of interference suppression in the adaptive antenna array is based on a weighted summation of beam patterns. For this representation we can get the system of equations. Herewith the system has a solution only if $M<N(N-$ numbers of the array elements, $M$ - number of the created nulls). 
Proposing method of synthesis beam pattern with nulls in specified directions do not control a directivity of the antenna array. LCMV beamformers provide some level of beam pattern in the interest signal direction [3] but this level not control by algorithm and depends on relative positions of the interest signal and jammers.

\section{REFERENCES}

[1] A.A. Erokhin, Yu.P. Salomatov, "Circular Antenna Arrays With Spatial Selection Of Interference", Russian Physics Journal, vol. 56, no. 8-2, pp. 122-124, 2013.

[2] Andreev A.G., Erokhin A.A., Salomatov Yu.P., Shtro P.V., "Radiation pattern zeros in the given direction", Electronic Equipment and Control Systems, no. 1, pp. 132-136, 2014.

[3] Wei Lui, Stephan Weiss, Wideband Beamforming: Concepts and Techiques, A John Wiley and Sons, Ltd., 2010, 286 p. 